\title{
Green and Inexpensive Method to Recover Bisphenol-A from Polycarbonate Wastes
}

\author{
Mir Mohammad Alavi Nikje, Mohammad Askarzadeh \\ Chemistry Department, Faculty of Science, Iran
}

\begin{abstract}
Hydroglycolysis of polycarbonate waste from used compact discs was developed to recover Bisphenol-A (BPA) as a valuable material. Experiments were done in glycerin and water as an alternative green solvent. The effect of solvent ratios has been studied to evaluate BPA recovery. The use of $30 \%$ aqueous glycerin (pbw) led to 93 and 100\% of BPA at reflux condition within 1.0 and 5.5 hours, respectively. Recovered BPAs were identified by spectroscopy methods and the results were compared with commercial sample data.
\end{abstract}

Keywords: Polycarbonate, bisphenol-A, recycling, hydroglycolysis.

\section{Introduction}

Polycarbonate is a well known category of condensed plastics containing unique properties which makes it be attractive for use in several areas, such as health, medical, electronic, electrical, automotive, leisure and safety applications ${ }^{[1]}$. The increasing amount of PC wastes from compact discs and plastic objects make their recycling development necessary ${ }^{[2]}$. The chemical recycling of polymer wastes has been gaining great attention as a common root in obtaining valuable products ${ }^{[3]}$. Depolymerization processes have been reported in order to decompose PC into its monomer, bisphenol A (BPA $)^{[4]}$, which is the main raw material for polycarbonate (PC) manufacturing. In this report, Pinero's research group investigated chemical recycling of $\mathrm{PC}$ in a semi-continuous lab-plant, using methanol and methanol-water mixtures as degradation reagents.

Oku et al. ${ }^{[5]}$ described the chemical conversion of $\mathrm{PC}$ to bis(hydroxyethyl) ether of BPA (BHE-BPA), which can be used as a diol for condensed polymers preparation. In this report, PC had been recycled using conventional heating method in the presence of ethylene glycol (EG) and catalytic amounts of $\mathrm{NaOH}$. In addition, the using of ethylene carbonate (EC) is necessary for preparation of BHE-BPA. Shwu-jer Chiu et al. ${ }^{[6]}$ investigated the effect of metal chlorides on thermal degradation of waste polycarbonate and found that $\mathrm{ZnCl}_{2}$ and $\mathrm{SnCl}_{2}$ can produce higher quantity of liquid products. In another report the depolymerization of PC in subcritical and supercritical toluene had been reported ${ }^{[7]}$. In the meantime, some other publications have been shown PC recycling, alone or blended with other polymers, which have their own merits and drawbacks ${ }^{[8-16]}$.

Developing of environmental friendly methods for PC recycling have been studied in recent years. Liu et al. ${ }^{[17]}$ reported methanolysis of polycarbonate (PC) to recover bisphenol A (BPA) and dimethyl carbonate (DMC) using an ionic liquid (n-butyl-3methylimidazolium chloride $([\mathrm{Bmim}][\mathrm{Cl}]))$. The BPA recovery and dimethylcarbonate formation yields were $95 \%$. The weight proportions of PC: $\mathrm{CH}_{3} \mathrm{OH}$ : [Bmim][Cl] have been set as $2: 3: 2$. The reaction temperature was $105{ }^{\circ} \mathrm{C}$ and reaction time was 2.5 hours $^{[17]}$. This research group also studied alkali-catalyzed methanolysis and hydrolysis of PC in a solvent in which PC can substantially dissolve such as $\mathrm{N}$-methyl-2-pyrrolidone, 1, 4-dioxane, tetrahydrofuran and BPA recovered in $94 \%$ yields ${ }^{[18]}$.

Taking into consideration the experiments already done by these authors on PC degradation ${ }^{[19]}$, it is presented a green, ecofriendly and convenient method in recovering BPA from CD wastes. The main goal of this work is to minimize the catalyst concentration, find eco-friendly method and avoid organic and toxic organic solvents.

\section{Experimental Part}

\section{Materials and methods}

Used CDs and DVDs for this study were collected from Qazvin city, crushed into the arbitrary sizes and shapes and washed with hot water and dried before being charged into the reactor. BPA, glycerin and sodium hydroxide were purchased from Merck and used as received without further purification. Water for performing of reactions was twice distilled. FT-IR spectra ( $\mathrm{KBr}$ pellet) were done by using a BRUKER Tensor 27 spectrometer. For this experiment, commercial and recovered BPAs $-\mathrm{KBr}$ pellets were used. ${ }^{1} \mathrm{HNMR}$ and ${ }^{13} \mathrm{CNMR}$ spectra were done using a BRUKER CRX 300 instrument using deuterated $\mathrm{CDCl}_{3}$ as a solvent and TMS as an internal standard and data compared by each other.

\section{General procedure}

Two-necked flask was charged with PC wastes $(2.7 \mathrm{~g})$, glycerin, water (solvent total weight $2.7 \mathrm{~g}$ ) and $\mathrm{NaOH}(2 \% \mathrm{pbw}$ based on all reactants) as the catalyst. The mixture was refluxed as denoted in the Table 1. All reaction sets were shown in Table 1. Separation and purification of bisphenol A were done by crystallization in water, besides filtration and drying. White BPA crystals were obtained, structurally characterized and the results were compared with pure sample data. In all reactions: solvent ratios were constant and set as 50:50 pbw.

Table 1. Effects of Glycerol/Water composition on BPA recovery yields.

\begin{tabular}{cccc}
\hline Experiment & $\begin{array}{c}\text { PC/Glycerin/ } \\
\text { Water }(\boldsymbol{p b w})\end{array}$ & $\begin{array}{c}\text { Reaction time } \\
\text { (hour) }\end{array}$ & $\begin{array}{c}\text { BPA recovery } \\
\text { yield }(\%)\end{array}$ \\
\hline 1 & $1.0 / 0.8 / 0.2$ & 0.25 & $\mathbf{8 6}$ \\
2 & $1.0 / 0.7 / 0.3$ & 0.50 & $\mathbf{8 6}$ \\
3 & $1.0 / 0.6 / 0.4$ & 1.00 & $\mathbf{9 3}$ \\
4 & $1.0 / 0.5 / 0.5$ & 1.50 & $\mathbf{9 5}$ \\
5 & $1.0 / 0.4 / 0.6$ & 1.75 & $\mathbf{9 9}$ \\
6 & $1.0 / 0.3 / 0.7$ & 5.50 & $\mathbf{1 0 0}$ \\
7 & $1.0 / 0.2 / 0.8$ & 6.50 & $\mathbf{9 5}$ \\
8 & $1.0 / 0.1 / 0.9$ & 7.50 & $\mathbf{9 2}$ \\
9 & $1.0 / 0.0 / 1.0$ & 35 & $\mathbf{7 0}$ \\
\hline
\end{tabular}




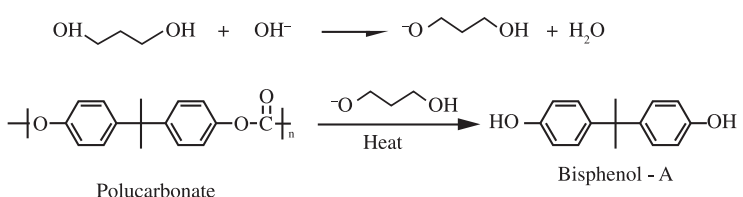

Mechanistic detail:
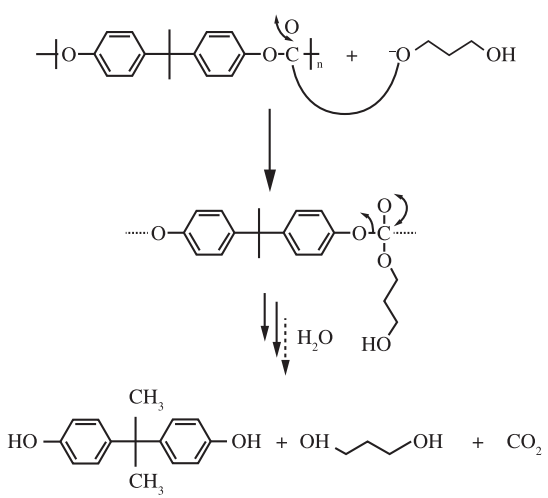

Figure 1. Reaction and mechanism for BPA recovering from polycarbonate.

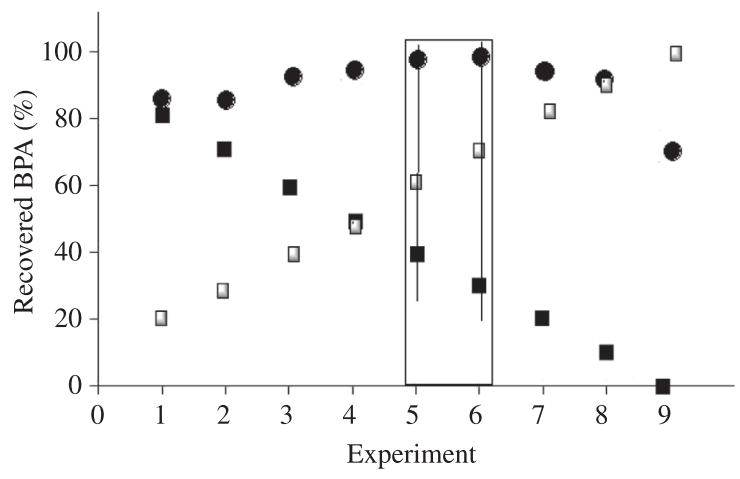

Area with high BPA recovery yields with adequate reaction times and solvent compositions

\section{- Glycerin BPA}

Figure 2. The effects of solvent composition on BPA recovering yields.
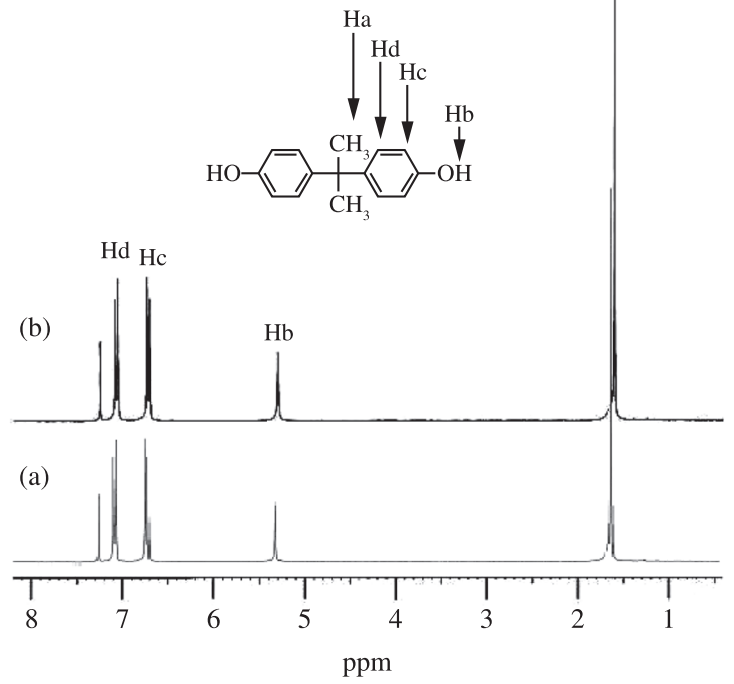

Figure 3. ${ }^{1} \mathrm{HNMR}$ spectra of commercial (a) and recovered (b) BPAs.

\section{Results and Discussion}

\section{Study on catalyst concentration}

Regarding to our experiments on recovering of BPA under microwave irradiation condition, data revealed high recovery yields in using $2.0 \%(p b w)$ of $\mathrm{NaOH}$ as the catalyst and for comparing data from MW and conventional heating methods, the base catalyst concentration was set as $2.0 \%(p b w)$ in all reactions ${ }^{[19]}$.

\section{Reaction scheme}

The Figure 1 shows the proposed reaction mechanism for PC depolymerization under alkali conditions in which BPA is recovered. The reaction first step is related to a Bronsted-Lowry acid-base reaction. The reaction is progressed by conversion of glycerin to the corresponded alkoxide ion and formation of negatively charged intermediate what hydrolyzes to BPA in the final step. The use of water as a solvent permits the action of hydroxide ion as a weak nucleophile, when compared with alkoxide ion (Table 1 and Figure 2).

\section{Solvent composition studies}

In order to find a green solvent composition containing high water content, the effects of ratios of water and glycerin were studied in BPA recovering yields. The data collected were shown in Table 1 and Figure 2, respectively. The results from experiments showed the highest recovering of BPA at 30\% glycerin $(p b w)$ solution (Experiment 6) as the solvent. In addition, by increasing of water content, the reaction takes place at prolonged times and BPA yields drop dramatically (Table 1 and Figure 2). The use of water alone provides the obtaining of BPA from PC much greener. The high reaction time is not technically feasible and the reaction needs using glycerin as a co-solvent. Due to aforementioned observations, the best and adequate conditions can be found between the

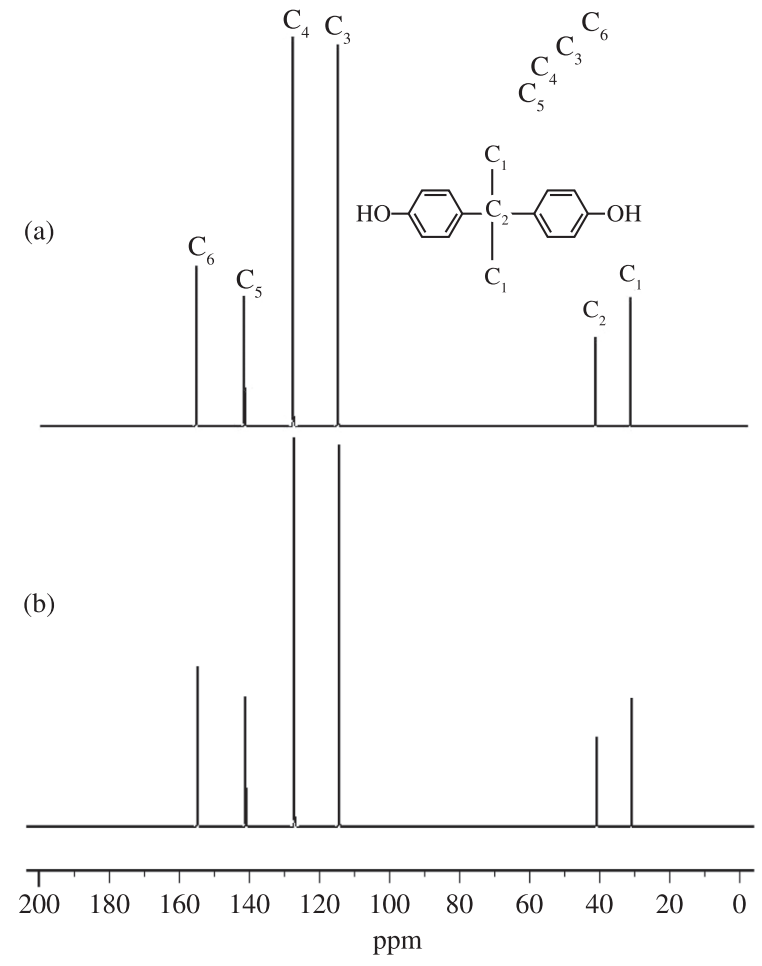

Figure 4. ${ }^{13} \mathrm{CNMR}$ spectra of commercial (a) and recovered (b) BPAs. 


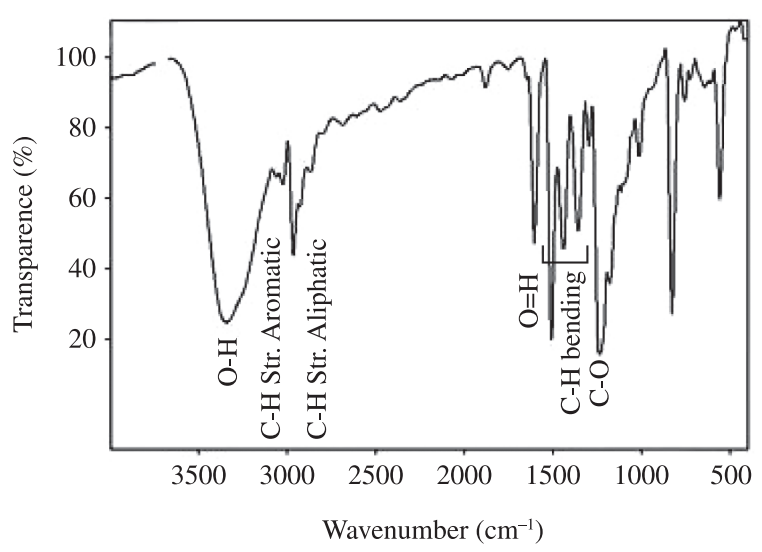

Figure 5. FTIR spectrum of recovered BPA.

Experiments 5 and 6. In order to study solvent composition effects on recovering rate, the concentration of base catalyst as well as CDs wastes were remained constant. One of the main advantages of our report in comparing with others is the using of maximum amounts of water as a green solvent.

\section{Recovered BPA chemical structure}

Recovered BPA was characterized and studied by spectroscopy methods in order to elucidate chemical structure and compare data by a commercial sample. The ${ }^{1} \mathrm{HNMR}$ spectrum of recovered BPA is presented in Figure 3. The peak at $1.53 \mathrm{ppm}$ corresponds to methyl hydrogen and the peak at $4.5 \mathrm{ppm}$ is relative to hydroxyl groups and the peaks at 6.5-7.0 ppm corresponds to aromatic hydrogen's. All ${ }^{1} \mathrm{HNMR}$ data was similar to data from a commercial sample that reveals successful recovering at this proposed experimental conditions.

The results and spectrum from ${ }^{13} \mathrm{CNMR}$ studies are shown in Figure 4. The peaks at 115 (C3), 127 (C4), 141 (C5) and 155 (C6) $\mathrm{ppm}$ are related to aromatic ring carbons. In addition, the signals at 31 and 41 ppm are attributed to methyl groups $(\mathrm{C} 1)$ and $\mathrm{C} 2$ carbons, respectively. FTIR spectrum (Figure 4) fully confirmed NMR data. The structure of recovered BPA can be confirmed by FTIR spectrum what is similar to commercial sample spectrum (Figure 5).

\section{Conclusion}

Water/glycerin mixture acts as a hydroglycolysis solvent in BPA recovering in a green, convenient and simple methodology. The results showed the performances of deporotonated glycerin in nucleophilic attached to carbonate functional groups of PC. The reaction was prolonged in absence of glycerin. This methodological proposal can be considered as an eco-friendly and green method for BPA recovering from CDs and DVDs wastes. In the meantime, strategy can overcome the disadvantages and drawbacks existed in traditional methods that apply organic solvents.

\section{References}

1. Brunelle, D. J. \& Korn, M. R. - "Advances in polycarbonates", American Chemical Society, Washington (2005). http://dx.doi. org/10.1021/bk-2005-0898

2. Statler, D. L. - "A mechanistic and modeling study of recycled and virgin flame retarded polycarbonate", Tesis doctorate, West Virginia University, Morgantown, West Virginia (2008).

3. Scheirs, J. - "Polymer recycling: Science, technology and applications", John Wiley \& Sons Ltd, England (1998).

4. Pinero, R.; Garcia, J. \& Cocero, M. J. - Green Chem., 7, p.380 (2005).

5. Oku, A.; Tanaka, S. \& Hata, S. - Polymer., 41, p.6749 (2000). http:// dx.doi.org/10.1016/S0032-3861(00)00014-8

6. Chiu, S. J.; Chen, S. H. \& Tsai, C. T. - Waste Manag., 26, p. 252 (2006). http://dx.doi.org/10.1016/j.wasman.2005.03.003

7. Pan, Z. Y.; Bao, Z. \& Chen, Y. X. - Chinese Chem. Lett., 17, p.545 (2006).

8. Lin, C. H.; Lin, H. Y.; Liao, W. Z. \& Dai, S. A. - Green Chem., 9, p.38 (2007). http://dx.doi.org/10.1039/b609638g

9. Jang, B. N. \& Wilkie, C. A. - Thermochim. Acta., 426, p.73 (2005). http://dx.doi.org/10.1016/j.tca.2004.07.023

10. Kim, J.; Gracz, H. S.; Roberts, G. W. \& Kiserow, D. J. - Polymer., 49, p.394 (2008). http://dx.doi.org/10.1016/j.polymer.2007.11.046

11. Jang, B. N. \& Wilkie, C. A. - Polym. Degrad. Stabil., 86, p.419 (2004). http://dx.doi.org/10.1016/j.polymdegradstab.2004.05.009

12. Bozi, J.; Czegeny, Z.; Meszaros, E. \& Blazso, M. - J. Anal. Appl. Pyrol., 79, p.337 (2007). http://dx.doi.org/10.1016/j.jaap.2007.01.001

13. Margon, V.; Agarwal, U. S.; Bailly, C.; Wit, G.; Van Kasteren, J. M. N. \& Lemstra, P. J. - J. Supercrit. Fluids., 38, p.44 (2006). http://dx.doi. org/10.1016/j.supflu.2005.11.007

14. Uyar,T.; Tonelli,A.E. \& Hacaloglu, J. - Polym. Degrad. Stabil.,91, p.2960 (2006). http://dx.doi.org/10.1016/j.polymdegradstab.2006.08.028

15. Fraisse, F.; Verney, V.; Commereuc, S. \& Obadal, M. - Polym. Degrad. Stabil., 90, p.250 (2005).

16. Jung, J. H.; Ree, M. \& Kim, H. - Catal. Today., 115, p.283 (2006). http://dx.doi.org/10.1016/j.cattod.2006.02.060

17. Liu, F.; Li, L.; Yu, S.; Lv, Z. \& Ge, X. - J. Hazard. Mater., 249, p.254 (2011).

18. Liu, F. S.; Li, Z.; Yu, S. T.; Cui, X.; Xie, C. X. \& Ge, X. P. - J. Polym. Environ., 17, p.208 (2009). http://dx.doi.org/10.1007/s10924-0090140-0

19. Alavi Nikje, M. M. - Polimery-W., 56, p.35 (2011).

Enviado: 06/03/12

Reenviado: $19 / 07 / 12$

Aceito: $23 / 07 / 12$ 